Variations tibétaines, Et autres...

\title{
Anna Jarry-Omarova, Genre du pouvoir et démocratie libérale en Mongolie. Analyse de l'échec du mouvement associatif des femmes, entre espace politique, nomadisme et ONG internationales
}

\section{OpenEdition \\ Journals}

Édition électronique

URL : https://journals.openedition.org/emscat/1944

DOI : 10.4000/emscat.1944

ISSN : 2101-0013

Éditeur

Centre d'Etudes Mongoles \& Sibériennes / École Pratique des Hautes Études

\section{Référence électronique}

«Anna Jarry-Omarova, Genre du pouvoir et démocratie libérale en Mongolie. Analyse de l'échec du

mouvement associatif des femmes, entre espace politique, nomadisme et ONG internationales », Études mongoles et sibériennes, centrasiatiques et tibétaines [En ligne], 42 | 2011, mis en ligne le 20 décembre 2011, consulté le 13 juillet 2021. URL : http://journals.openedition.org/emscat/1944 ; DOI https://doi.org/10.4000/emscat.1944

Ce document a été généré automatiquement le 13 juillet 2021.

(c) Tous droits réservés 


\section{Anna Jarry-Omarova, Genre du pouvoir et démocratie libérale en Mongolie. Analyse de l'échec du mouvement associatif des femmes, entre espace politique, nomadisme et ONG internationales}

\section{RÉFÉRENCE}

Thèse de doctorat soutenue le 2 juillet 2010, à l'Ecole des Hautes Etudes en Sciences Sociales (Centre d'Etudes des Mouvements Sociaux). Directeur de thèse : Djallal G. Heuzé, Directeur de Recherche CNRS/EHESS Consultable en ligne : http://hal.archives-ouvertes.fr/tel-00545781_v1/

1 Ma thèse de doctorat ${ }^{1}$ a cherché à révéler les mécanismes explicatifs du net recul de la présence des femmes au sein de l'espace du pouvoir en Mongolie, depuis l'adoption de sa constitution démocratique en 1992. Les femmes composaient pourtant entre le quart et le tiers des représentantes à l'époque communiste, elles ont également participé aux manifestations populaires de transition démocratique. Elles ont surtout constitué un mouvement associatif féminin dont l'objectif majeur était justement la promotion des femmes en politique. Une enquête de terrain de 24 mois a permis de réaliser plus de 130 entretiens avec des militantes politiques et associatives, tant en ville qu'à la campagne. Ces rencontres formelles ont été complétées par des observations du quotidien lorsque j'étais accueillie chez certaines d'entre elles pour des séjours de courte durée. J'ai également assisté à de nombreuses réunions de travail au sein des associations et partis politiques. Il s'agissait d'observer leurs stratégies, individuelles et/ou collectives, à 
l'aide de deux outils conceptuels : celui du «capital», social et politique, et celui d'« espaces » sociaux, lors des campagnes électorales.

2 En effet, "[la] capacité de mobilisation [des électeurs-trices pour un candidat spécifique] est liée à la possession de capital politique soit à titre personnel, soit par délégation [de la part du Parti politique par exemple]. [...] [I]l y a accumulation de forces, de capital politique, c'est à dire de réputation" [souligné par nous]. L'accumulation de capitaux sociaux est ainsi transformable en capital politique afin d'accéder à l'espace du pouvoir. La science politique travaille par exemple les «multiples capitaux» de candidats politiques (Achin et Dorlin 2007): le capital intellectuel, familial, matériel, symbolique (proche de la notion de "gloire héroïque »), etc. De cette accumulation, de ce capital et de lui seul, dépend l'entrée dans l'espace du pouvoir. Il peut alors être transmis en héritage (d'un leader vers un dauphin), mais il peut également se constituer - souvent de haute lutte individuelle et/ou collective - et on a alors besoin de l'entretenir. Le pendant de l'accumulation est son délitement, comme le montre Boris Pétric dans ses recherches sur l'accès au pouvoir en Ouzbékistan.

3 La première partie de la thèse explore l'espace public-politique. Elle expose la reconstruction exclusivement masculine, virile, des jeunes partis politiques et du nouvel espace de débat démocratique (médias et assemblées). Avec une comparaison parfois surprenante de la situation française, on découvre que le soutien des leaders va en priorité aux jeunes hommes, ce qui signifie que les femmes sont largement moins " héritières de capital politique » que les jeunes hommes. Le second chapitre étudie l'impact de la libéralisation de l'économie sur la participation au politique. L'appauvrissement économique est beaucoup plus marqué pour les femmes, c'est-à-dire qu'elles bénéficient d'un accès bien moindre aux capitaux financiers que les hommes. Outre le fait que les femmes se replient alors sur des activités domestiques (comme le traitement des produits de l'élevage) qui les éloignent des espaces publics et de pouvoir, les campagnes électorales sont devenues payantes et rares sont les femmes qui engagent une fortune, qu'elle ne possèdent pas et dont elles ne sont pas assurées du bénéfice. Surtout, alors que le personnage de Gengis Khan se trouve revalorisé au niveau national (Aubin 1993), l'adoption d'une économie de marché a promu l'image du guerrier économique, qui peut aussi accéder au pouvoir. C'est une image à laquelle les femmes ne " collent pas », elles qui prônent au contraire des notions de solidarité et de répartition des richesses : cela les exclut de l'espace des luttes politiques. Le troisième chapitre étudie le "capital intellectuel », que les femmes possèdent largement puisqu'il est de notoriété publique qu'elles sont plus diplômées que les hommes. L'idéologie de genre en Mongolie est intéressante, en ce que l'identité de "femme " intègre une dimension de "femme avisée ", intelligente et de bon conseil, ce qu'avaient déjà démontré R. Hamayon et N. Bassanov (1973) dans leur analyse des contes mongols. La situation est tout autre dans l'idéologie de genre en France, où les contes pour enfants mettent en scène des jeunes filles passives, soumises et sans parole (la Belle aux Bois Dormant, Blanche Neige, etc.). La thèse montre cependant comment ce capital intellectuel, dont les Mongoles sont si fières, est plus un instrument aux mains des leaders politiques qu'il n'est un atout valorisable par les intéressées. On vient les chercher et on les place, mais dès qu'elles deviennent trop revendicatrices, on les écarte. On constate enfin qu'elles n'ont pas accès à la parole publique, ce qui est pourtant essentiel au sein de l'espace public et du pouvoir, surtout en démocratie. Ceci 
avait déjà été montré par R. Hamayon (1979) : la mise en place d'un régime politique démocratique n'a donc pas permis d'évolution vers l'égalité entre hommes et femmes en Mongolie, on constate même un certain recul dans ce domaine.

4 La seconde partie de la thèse explore le "genre de la culture nomade ", tant dans les pratiques concrètes que symboliques au sein de la famille, l'élevage pastoral, la gestion des véhicules et enfin le système des échanges de biens et de services. Bien que présentant des pratiques exceptionnellement égalitaires du point de vue du genre, comme celle de la mobilité des femmes à cheval (Aubin 1986), la société mongole n'en demeure pas moins patriarcale. Il en est de même pour l'espace domestique (la yourte et l'espace délimité par la ronde du chien de garde alentour), où les femmes assument des «responsabilités» à la teneur obligatoire et qui ne sont pas source de reconnaissance sociale, ce qui diffère de la notion de "pouvoir ». Elles participent aux prises de décision, mais demeurent sous la domination masculine symbolisée par l'espace nord de la yourte, occupé exclusivement par les hommes et qui leur permet de contrôler l'ensemble de la yourte, espace féminin sud-est compris.

5 Le sixième chapitre étudie la circulation des biens et services, dimension essentielle de la vie sociale mongole grâce au tissage des solidarités entre urbains et ruraux (Sneath 1993 et 2006, Bikales, Khurelbaatar et Schelzig 2000). L'anthropologie, la première, avec les travaux de Marcel Mauss sur le don et le contre-don, montre combien «[...] la question première qui se pose aux hommes [est] la question politique qui n'est autre que l'autre face de la question du don, celle de savoir qui sont les amis et qui sont les ennemis. [Ainsi,] le don et le politique coïncident. » (Caillé 2000, p. 84, 128). Le réseau et, à travers lui, la circulation des dons qui obligent aux contre-dons, permet la constitution d'un capital social qui va se transformer en un " capital politique ». Cette dynamique semble essentielle à $P$. Bourdieu : «L'existence d'un réseau [est] le produit de stratégies [...] orientées vers l'institution ou la reproduction de relations sociales directement utilisables [...] produisant la connaissance et la reconnaissance mutuelles. » (Bourdieu 1980, p. 2). La reconnaissance permet "une dépossession » (Bourdieu 2005, p.55) volontaire de la part du citoyen de son capital politique, décisionnel, vers une élite politique. Les uns fournissent aide et cadeaux, les autres peuvent effectuer des contre-dons matériels et/ou symboliques. Localement, le contredon symbolique par excellence est bien l'élection politique de celui qui cumule le plus de «capital réputationnel» (Bourdieu, 2005, p. 55). D'autant plus dans une société anciennement communiste, où le régime était supposé avoir effacé les distinctions de richesse matérielle (Humphrey 1980, Pétric 2002).

La thèse explore la dimension du genre au sein de ces échanges, à travers l'étude de la circulation de trois catégories de biens: l'entraide de tout ordre, les offrandes strictement féminines de deel (habit tratitionnel mongol, masculin et féminin) et la consommation de vodka. Ces échanges ne confèrent pas les mêmes bénéfices aux hommes et aux femmes, ce qui explique encore pourquoi elles n'accèdent pas au pouvoir. Les femmes sont obligées de "donner », mais elles ne "reçoivent » pas. À la façon de la "Mère ", figure puissante de l'idéologie de genre mongole, leur don est sacrificiel, il y va de leur identité de "femme bonne » (sain emegtei). Par exemple, si une jeune fille ne s'y conforme pas, elle va alors être considérée comme une "mauvaise Mongole » (muu Mongol hün). Les femmes ne sont donc pas en droit de s'attendre à un contre-don, a fortiori au contre-don symbolique de l'élection politique. Elles sont donc exclues du système permettant d'accéder aux mandats électifs. 
7 La troisième partie de la thèse finit par expliquer l'échec du mouvement associatif des femmes. Leur première stratégie a été de tenter de constituer un "capital féminité », avec pour principal argument que « les femmes seraient meilleures que les hommes": plus éduquées, moins corrompues, moins souvent alcooliques, etc. La seconde fut de s'appuyer sur l'aide internationale, tant sur le plan financier que symbolique (en citant les pays occidentaux comme des exemples à suivre, entre autres références symboliques à ces pays et organisations internationales partenaires). Cette double stratégie n'a pas fonctionné, la seconde ayant anéanti la première: les leaders nationaux n'ont en effet pas accepté cette sorte "d'ingérence extérieure par les femmes ", ce qui, au contraire des objectifs du mouvement associatif des femmes, a provoqué un renforcement de la domination masculine au nom de la Nation.

8 En conclusion de la thèse, et face à cet ensemble de dynamiques de résistances masculines, il nous apparaît que le seul véritable espace social qui demeure privé est non pas la famille mais plutôt l'espace du pouvoir. Femmes et hommes occupent l'ensemble des espaces sociaux, y compris politique, et ce sont les hommes qui les contrôlent, quels que soient les capitaux sociaux que les femmes peuvent détenir et mobiliser. Les femmes sont bien absentes - et tenues à l'écart - de l'espace restreint du pouvoir, à l'instar de toutes les minorités politiques. Celui-ci demeure fermé, secret et auto-reproduit. Et l'instauration d'une démocratie n'a non seulement pas permis sa " publicisation », mais semble plutôt avoir renforcé sa " privatisation virile ».

\section{BIBLIOGRAPHIE}

Achin C. et Dorlin E.

2007 «J'ai changé, toi non plus. » La fabrique d'un-e présidentiable : Sarkozy/Royal au prisme du genre, Mouvements (http://www.mouvements.info/J-ai-change-toi-non-plus.html).

Aubin F.

1986, « L'art du cheval en Mongolie » in Production Pastorale et Société, 19, pp. 129-189.

1993 Renouveau gengiskhanide et nationalisme dans la Mongolie postcommuniste, Cahiers

d'études sur la méditerranée orientale et le monde turco-iranien, 16, pp. 137-203.

Bikales B., Khurelbaatar C. et Schelzig K.

2000 The Mongolian informal sector : Survey results and analysis, in USAID 'Economic Policy Support' project (Ulaanbaatar : Development Alternatives Inc).

Bourdieu P.

1980 Le capital social : Notes provisoires, Actes de la Recherche en Sciences Sociales, 31, pp. 2-3. [2000] 2005 Propos sur le champ politique (Lyon, Presses Universitaires de Lyon).

Caillé A.

2000 Anthropologie du don. Le tiers paradigme (Paris, Desclee de Bourwer, Sociologie économique).

Hamayon R.

1979 Le pouvoir des hommes passe par la langue des femmes. Variations mongoles sur le duo de la légitimité et de l'aptitude, L'Homme, 19, pp. 109-139. 
Hamayon R. et Bassanov N.

1973 De la difficulté d'être une belle-fille, Cahiers d'Etudes Mongoles et Sibériennes, 4, pp. 7-73.

Humphrey C.

1980 Introduction, in Vainshtein, S. I., Nomads of South Siberia : The Pastoral Economies of Tuva

(Cambridge, Cambridge University Press).

Pétric B.

2002 Pouvoir, don et réseaux en Ouzbékistan post-soviétique (Paris, Presses Universitaires de France, Partage du savoir).

Sneath D.

1993 Social relations, networks, and social organization in post-socialist rural Mongolia, Nomadic Peoples, 33, pp. 193-207.

2006 Transacting and enacting : Corruption, obligation and the use of monies in Mongolia, Ethnos, 71, pp. 89-112.

\section{NOTES}

1. La collecte des données sur le terrain a bénéficié de trois bourses: Bourse d'étude du Ministère des Affaires étrangères français, dans le cadre du Programme de coopération de recherche scientifique entre la France et la Mongolie (1998-1999 puis 1999), bourse Lavoisier du Ministère des Affaires étrangères français (2000) et bourse « Aire culturelle » du Ministère de la Recherche (2000). 